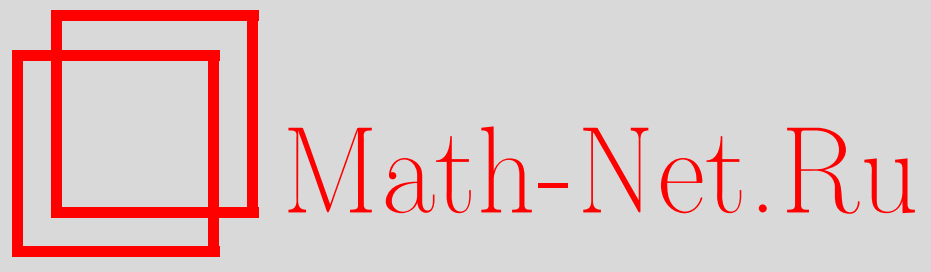

Е. А. Большакова, Непримитивные $n$-мерные параллелоэдры первого типа: комбинаторика и символы, УМH, 2006, том 61, выпуск 3, 167-168

DOI: https://doi.org/10.4213/rm1748

Использование Общероссийского математического портала Math-Net.Ru подразумевает, что вы прочитали и согласны с пользовательским соглашением http://www . mathnet.ru/rus/agreement

Параметры загрузки:

IP : 54.198 .64 .247

26 апреля 2023 г., 16:19:00

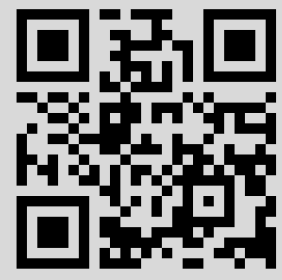




\section{Непримитивные $n$-мерные параллелоэдры первого типа: комбинаторика и символы}

\section{Е. А. Большакова}

1. Задачей настоящего сообщения является описание одного метода кодировки комбинаторной структуры параллелоэдра первого типа (подробное изложение результатов предполагается опубликовать в Чебышёвском сборнике). С основными понятиями теории параллелоэдров можно познакомиться по статье [1], а далее - по цитированной в ней литературе. Понятия и факты теории графов, если они не определены в тексте, см. в [2]. Всю необходимую информацию о зоноэдрах можно найти, например, в [3].

В $N$-мерном $\left(N=\frac{n(n+1)}{2}\right)$ евклидовом пространстве коэффиииентов квадратичных форм от $n$ переменных выделяется выпуклый замкнутый конус положительности $\mathbb{K}^{N}$. Внутренним точкам этого конуса отвечают положительно определенные квадратичные формы, а граничным - неотрицательно определенные квадратичные формы ранга меньшего, чем $n$. Конус $\mathbb{K}^{N}$ разбивается на области так называемых арифметических $L$-типов [4]. Эти области суть выпуклые конечногранные полиэдральные конусы, к каждому из которых естественно причислять и его границу, в том числе экстремальные лучи. Квадратичная форма, соответствующая произвольной фиксированной точке, лежащей на экстремальном луче, называется реберной формой соответствующей области типа. Из [1] следует, что любой параллелоэдр есть взвешенная сумма Минковского параллелоэдров, соответствующих реберным формам той области типа, которой принадлежит соответствующая ему квадратичная форма.

Среди всех областей $L$-типа наиболее простое описание имеет область первого тu$n a$, при любом $n$ представляющая собой симплициальный конус. Ей принадлежат квадратичные формы, представимые следующей суммой:

$$
f\left(x_{1}, \ldots, x_{n}\right)=\sum_{0 \leqslant i<j \leqslant n} \lambda_{i j}\left(x_{i}-x_{j}\right)^{2}, \quad \text { где } \quad \lambda_{i j} \geqslant 0, \quad \sum \lambda_{i j}^{2}>0 .
$$

Здесь формально полагается $x_{0}=0$. Параллелоэдры, соответствующие квадратичным формам ранга 1 от $n$ переменных суть отрезки. Таким образом, все параллелоэдры первого типа суть суммы Минковского отрезков, т.е. зоноэдры.

Поскольку параллелоэдры, соответствующие точкам одной и той же открытой грани области $\Delta_{1}$, отличаются друг от друга только метрическими характеристиками, достаточно описать структуру одного параллелоэдра из этого множества, отвечающего “эталонной” квадратичной форме, в разложении $(*)$ которой каждый коэффициент $\lambda_{i j}$ равен либо 0, либо 1.

Такие квадратичные формы описываются символом Рышкова: для квадратичной формы $f\left(x_{1}, \ldots, x_{n}\right)$ это граф с вершинами $v_{0}, v_{1}, \ldots, v_{n}$, и ребро $\left(v_{i}, v_{j}\right)$ в нем есть тогда и только тогда, когда в разложении $(*)$ формы $f$ коэффициент $\lambda_{i j}$ равен 1 .

2. ОПреДЕЛЕНИЕ. Назовем $k$-вырезом $(0 \leqslant k \leqslant n)$ в графе $G$ всякое множество $R^{k}$ ребер графа $G$ такое, что его удаление из графа увеличивает количество компонент связности на $k$, а удаление всякого множества $R^{\prime} \subset R^{k}, R^{\prime} \neq R^{k}$ увеличивает количество компонент связности менее, чем на $k$.

ОПределение. Допустимой ориентацией $k$-выреза $R^{k} \subset E$ в графе $G=(V, E)$ будем называть всякое придание ориентации всем ребрам $x \in R^{k}$, обладающее свойством: невозможно так ориентировать ребра $y \in E \backslash R^{k}$, чтобы в графе $G$ образовался орцикл, имеющий хотя бы одно ребро $x_{0} \in R^{k}$.

ОПредЕлЕНиЕ. Назовем $k$-остатком графа $G=(V, E)$ граф $G_{k}=\left(V, E \backslash R^{k}\right)$, где $R^{k}$ - некоторый $k$-вырез в графе $G$.

\footnotetext{
${ }^{1}$ Работа выполнена при финансовой поддержке INTAS, грант № 03-51-5070.
} 
Теорема 1. Пусть Я - параллелоэдр первого типа, соответствующий символу $G, a F-$ его гранъ размерности $k \leqslant n$. Тогда грань $F$ параллельно конгруэнтна параллелоэдру, отвечающему некоторому $(n-k)$-остатку графа $G$. Обратно, если $G_{n-k}$ - это $(n-k)$-остаток $(1 \leqslant k \leqslant n)$ символа $G$ и $\mathscr{P}_{*}$ - соответствующий ему параллелоэдр, то в параллелоэдре Я есть семейство $k$-мерных граней, параллельно конгруэнтных параллелоэдру $\mathscr{P}_{*}$.

Отклоняющим вектором грани $\mathscr{F}$ с центром симметрии $O_{F}$ параллелоэдра $\mathscr{P}$ с центром симметрии $O_{P}$ будем называть вектор $x=\overrightarrow{O_{P} O_{F}}$.

Теорема 2. Пусть $\mathscr{P}$ - параллелоэдр первого типа, $G$ - его символ, $R^{n-k}-$ $(n-k)$-вырез в графе $G$ и $G_{n-k}$ - соответствуюший $(n-k)$-остаток, $\mathscr{P}_{*}$ - параллелоэдр, отвечающий символу $G_{n-k}$. Среди $k$-мерных граней параллелоэдра Я尹 , параллельно конгруэнтных параллелоэдру $\mathscr{P}_{*}$, тогда и только тогда есть грань с отклоняющим вектором $x$, когда существует такал допустимая ориентация $(n-k)$-въреза $R^{n-k}$, что $x=\sum_{\left(v_{i} \rightarrow v_{j}\right) \in R^{n-k}}\left(e_{j}-e_{i}\right)$, где $\left(v_{i} \rightarrow v_{j}\right)$ - ребра ориентированного $(n-k)$-выреза $R^{n-k}$, а $e_{1}, \ldots, e_{n}-$ координатные векторы.

3. ОПределЕниЕ. Пусть $G_{1}=\left(V_{1}, E_{1}\right)$ и $G_{2}=\left(V_{2}, E_{2}\right)$ - два графа. Отображение $\varphi: E_{1} \rightarrow E_{2}$ множества ребер первого графа в множество ребер второго графа сохраняет простой цикл $C$ графа $G_{1}$, состоящий из ребер $x_{1}, \ldots, x_{k}$, если ребра $\varphi\left(x_{1}\right), \ldots, \varphi\left(x_{k}\right)$ образуют, возможно в другом порядке, простой цикл в графе $G_{2}$.

ОПРЕДЕЛЕНИЕ. Назовем два графа $G_{1}=\left(V_{1}, E_{1}\right)$ и $G_{2}=\left(V_{2}, E_{2}\right)$ ииклически неразличимыми, если между ребрами одного и другого графа установлено взаимно однозначное соответствие $\varphi: E_{1} \rightarrow E_{2}$, которое сохраняет все простые циклы графа $G_{1}$, и притом обратное отображение $\varphi^{-1}: E_{2} \rightarrow E_{1}$ сохраняет все простые циклы графа $G_{2}$. Пример неизоморфных циклически неразличимых графов см. в [2; рис. 13.3].

Теорема 3. Пусть $G_{1}$ и $G_{2}$ - два символа первого типа, $f_{1}$ u $f_{2}$ - coответствующие им квадратичные формы первого типа, а $\mathscr{P}_{1}$ и $\mathscr{P}_{2}$ - соответствующие параллелоэдры. Тогда следующие три утверждения эквивалентны.

1. Параллелоэдры $\mathscr{P}_{1}$ и $\mathscr{P}_{2}$ комбинаторно эквивалентны.

2. Символы $G_{1}$ и $G_{2}$ ииклически неразличимы.

3. Формы $f_{1}$ и $f_{2}$ челочисленно унимодулярно эквивалентны.

Импликация $3 \Rightarrow 1$ практически очевидна, доказательство импликации $1 \Rightarrow 2$ несложно. Импликации $2 \Rightarrow 3$ автором дано два доказательства, опирающихся на теорему Уитни [5]. Первое из них проще, зато второе попутно положительно решает вопрос о возможности согласованной с отображением $\varphi$ ориентации двух циклически неразличимых графов. Формулировку и нетривиальную историю доказательства теоремы Уитни также см. [6; с. 255-258].

ЗАмечАние. Теорема 3 также положительно решает вопрос об эквивалентности понятий комбинаторного типа и $L$-типа параллелоэдров в частном случае (непримитивных параллелоэдров первого типа).

Автор очень благодарен своему научному руководителю С. С. Рышкову.

\section{Список литературы}

[1] С. С. Рышков, Е.А. Большакова, Изв. РАН. Сер. матем., 69:6 (2005), 187-210. [2] Ф. Харари, Теория графов, Мир, М., 1973. [3] P. McMullen, Trans. Amer. Math. Soc., 159 (1971), 91-109. [4] С. С. Рышков, Е. П. Барановский, УМH, 34:4 (1979), 3-63. [5] H. Whitney, Amer. J. Math., 55:2 (1933), 245-254. [6] А. А. Зыков, Основы теории графов, Вузовская книга, М., 2004.

Е. А. Большакова (Е. А. Bol'shakova)

Московский государственный университет им. М. В. Ломоносова
Представлено В. М. Бухштабером Принято редколлегией 19.04.2006 